\title{
Kapitulationskonzepte im Alpen-Adria-Raum 1945
}

Die militärischen und politischen Abläufe im Mai 1945 in der Operationszone Adriatisches Küstenland (Friaul, Julisch Venezien, Triest, Istrien), in Kärnten und Slowenien weichen in ihren Grundzügen beträchtlich von der Situation in anderen Frontabschnitten ab, zeigen aber zugleich in besonders scharfer Ausprägung Entwicklungen auf, die anderswo zum Teil nur in geringfügigen Ansätzen sichtbar wurden oder aber ausblieben. Es scheint, soweit bekannt, in diesen Gebieten einmalig gewesen zu sein, daß die politische und allgemeine Verwaltungsinstanz über die militärischen Instanzen hinweg entschied und eine neue Front aufbaute. Zum andern vollzog sich aber in diesem Bereich, den man in etwa mit dem Alpen-Adria-Raum gleichsetzen kann, im militärischen Gesamtapparat von unten weit nach oben fortschreitend eine so nachhaltige Veränderung der Befehlsstrukturen - die man als eine Art Auflösung bezeichnen könnte - daß sich höhere militärische Führungsebenen in zunehmendem Maße isoliert sahen. Dieser in den mittleren Rängen der Basis einsetzende Veränderungsprozeß scheint politisch motiviert gewesen zu sein und führte zur Entwicklung von verschiedenen Varianten der Kapitulation, ja überhaupt zu grundlegend voneinander divergierenden Konzeptionen der Kampfeinstellung. Zugleich aber verlief dieser Veränderungsprozeß nicht einheitlich, sondern er führte auch zu jener scharfen Polarisierung der Kräfte, welche letztlich die Verlängerung des Krieges bis zum 15. Mai mit sich brachte.

$\mathrm{Da}$ eine Reihe von bisher unbekannten Dokumenten aufgefunden worden sind, kann die Klärung dieser komplexen Sachlage nunmehr mit größerer Sicherheit möglich sein, denn besonders hinsichtlich der nordostitalienischen Front bestand bisher eine nicht unbeträchtliche Forschungslücke.

In Kärnten, im Süden "vorgelagert«, befanden sich im April 1945 die Heeresgruppe C (OB Südwest, v. Vietinghoff) und die Heeresgruppe E (OB Südost, Löhr). Der Isonzo bildete die Grenze zwischen beiden. Am 25. April 1945 unterstellte der Wehrmachtführungsstab B das LXXXXVII. Armeekorps von General Kübler (188. Geb. Div., 237. Inf. Div., Reste der 392. kroatischen Div.) dem OB Südost, um der Bedrohung Fiumes durch die rasch vorankommende 4. Jugoslawische (Dalmatinische) Armee entgegenzuwirken. Der größte Teil des Korps wurde deshalb im Raum Fiume konzentriert. Zum gleichen Zeitpunkt war der Höhere SS- und Polizeiführer im Oberabschnitt Alpenland und in der Provinz Laibach (Operationszone Adriatisches Küstenland), Obergruppenführer Rösener, zum Kommandeur der rückwärtigen Gebiete der Heeresgruppe E ernannt worden. Der später von Löhr befohlene und aus dem Raum südlich Laibach vorgetragene Entsatzversuch für das schließlich im Raum Fiume - Villa del Nevoso eingekesselte LXXXXVII. Korps blieb bereits nach geringen Anfangserfolgen liegen ${ }^{1}$.

Im Zuge dieser Ereignisse entwickelte sich in Friaul und Julisch Venezien ein beträchtliches Vakuum, das von den Verbänden kaum gefüllt werden konnte, die dem Höheren SS- und Polizeiführer der Operationszone Adriatisches Küstenland (ausschließlich der Provinz Laibach), Gruppenführer Odilo Globocnik, unterstanden. Es waren dies die Verbände der Ordnungspolizei, sodann die 24. SS-Gebirgs-Karstjäger-Division (Brigadestärke), die zurückgebliebenen Rekrutenkompanien der 188. Gebirgsdivision, Marine-Flak-Einheiten, fremdvölkische Verbände Globocniks sowie die ihm unterstellten Cetnik-Einheiten und der Verband „Kosakenland in Norditalien «, der in drei Brigaden gegliedert war, schließlich noch italienische Einheiten, wie etwa das I. Bataillon des 8. Reggimento Bersaglieri "Bruno Mussolini« (zu- 
letzt umgegliedert zum Italienischen Küsten- und Festungsbataillon XV), mehrere Bataillone der Decima Mas Borgheses u.a. ${ }^{2}$.

Der Abzug der meisten Einheiten des LXXXXVII. Korps und die dadurch erfolgte "Verdünnung« der Kräfte in Friaul und Julisch Venezien wurde vom Stab Globocniks sehr kritisch beurteilt, zumal sich zu diesem Zeitpunkt weiter westlich in Venezien die Auflösung der Heeresgruppe $\mathrm{C}$ schon deutlich abzeichnete ${ }^{3}$. Der Reichsgau Kärnten selbst hatte im April 1945 als Unterbringungs- und Versorgungsraum sowohl für die Heeresgruppe Süd (ab 30. April Heeresgruppe Ostmark genannt), die Heeresgruppe $E$ und die Heeresgruppe $C$ wegen des Näherrückens der Fronten zunehmend an Bedeutung gewonnen ${ }^{4}$. Auch der Reichsstatthalter von Kärnten und Oberste Kommissar in der Operationszone Adriatisches Küstenland, Rainer, beobachtete die Situation genau. Als am 29. April die Kapitulationserklärung der Heeresgruppe $C$ unterschrieben wurde, trachtete er danach, die Rolle Kärntens als Unterbringungsraum zu klären. Sein Ansuchen, das er schon an diesem Tag an den Wehrmachtführungsstab B richtete, dürfte sich wohl kaum mit der Aufteilung Ostkärntens zwischen den Heeresgruppen Süd und E beschäftigt haben, sondern berücksichtigte vielmehr die bevorstehende Kapitulation der Heeresgruppe C. Die Antwort von Generalleutnant Winter ging zwar am 30. April (16.00 Uhr) beim Stellvertretenden Generalkommando XVIII. AK (General Ringel) ein, erreichte aber laut Eingangsstempel Rainers Kanzlei erst am 4. Mai: "Ihren Antrag, das Land Kärnten geschlossen in den Befehlsbereich des OB Südost zu nehmen, habe ich nochmals eingehend geprüft. Ich kann ihm jedoch leider nicht entsprechen, da taktische und operative Gründe eine andere Abgrenzung über militärische Befehlsbereiche unbedingt erfordern. Ihr Winter. ${ }^{5} \mathrm{Am}$ 4. Mai allerdings war diese Entscheidung durch den Gang der Ereignisse in Oberitalien überholt. Rainer jedenfalls wollte von Anfang an gerade wegen des Zusammenbruches der Heeresgruppe $C$ den Reichsgau Kärnten als integrierenden Bestandteil des Wirkungsbereiches der Heeresgruppe E sehen. Am 4. Mai noch gehörte aber zum rückwärtigen Gebiet der Heeresgruppe E lediglich das Gebiet Unterdrauburg Kamm der Saualpe - Mühlen - Karlnock - Villach (ausschließlich) bis Reichsgrenze südlich ${ }^{6}$.

Bei der Auslegung des Textes der Kapitulationserklärung der Heeresgruppe $\mathrm{C}^{7}$ zeigte sich auf alliierter Seite sogleich die Tendenz, den Reichsgau Kärnten in diese Teilkapitulation einzubeziehen, was jedenfalls von den alliierten Sendern auch in Kärnten und Friaul mit Nachdruck verbreitet wurde. Rainer informierte die Bevölkerung erst am 3. Mai über den Rundfunk, machte aber nur wenige Andeutungen vom alliierten Durchbruch über den Po und von der Teilkapitulation der Heeresgruppe C; allerdings betonte er mit Nachdruck, daß der Reichsgau Kärnten in diese Kapitulation nicht einbezogen sei. Dabei erwähnte Rainer, daß Kesselring ihn zu diesen Mitteilungen ermächtigt habe ${ }^{8}$.

Mittlerweile hatten die Vorausabteilungen des V. britischen Korps (Lt. Gen. Keightley) am 1. Mai Pordenone und Udine erreicht, jene des XIII. britischen Korps (Gen. Harding) am Nachmittag des gleichen Tages Monfalcone. Beide Korps gehörten zur britischen 8. Armee (Gen. McCreery). Der weitere britische Vormarsch in Richtung Triest erwies sich dagegen wegen des massiven jugoslawischen Widerstands als schwierig, zumal ja inzwischen die Diskussion um die angloamerikanischen und jugoslawischen Kompetenzen in Julisch Venezien und Triest voll entbrannt war. Die 4. Jugoslawische Armee hatte mit einem Teil ihrer Einheiten das deutsche LXXXXVII. Korps im Raum Fiume - Villa del Nevoso eingeschlossen - es kapitulierte erst am 7. Mai -, die 29. und die 15. Division der 4. Jugoslawischen Armee wandten sich aber nach der Einschließung sofort nach Norden in Richtung Laibach und gewannen gegen Röseners 17. und 19. SS-Polizeiregiment ständig an Boden, so 
daß sie einerseits bald vor allem am rechten Flügel Kontakt mit den südöstlich Laibach eingesetzten italienisch-kommunistischen Partisanenstreitkräften hatten und sich andererseits im Raum Mirna Pec an den linken Flügel der 3. Division der 2. Jugoslawischen Armee anschließen konnten.

Zum Zeitpunkt dieser Entwicklung hatte die Hauptmasse der 4. Jugoslawischen Armee den Raum Fiume umgangen und war unbeirrt mit massiven Kräften weiter in Richtung Triest vorgestoßen. Dort hatte sich inzwischen eine in politischer und militärischer Hinsicht sehr komplexe Lage ergeben. Globocnik hatte am 30. April seine Stäbe aus Triest abgezogen, zugleich auch das 1. Regiment Milizia Difesa Territoriale. Gleichzeitig waren auch andere Einheiten in den Sammelraum nördlich Udine abgesetzt worden: Die 1. Dinarische Division (Cetniks) und das 1. Regiment Slovenski narodni varnostni zbor (Domobranci) aus Aidussina, das 10. SS-Polizeiregiment und das 4. Regiment Milizia Difesa Territoriale aus Görz, der »Führungsstab Bandenkampf« mit Teilen der 24. SS-Freiwilligen-Gebirgsdivision Karstjäger und das A-Bataillon der 7.SS-Freiwilligen-Gebirgsdivision aus Gradisca; letztere Einheit wurde in Richtung Laibach verlagert.

Diesen Einheiten standen zuerst folgende Partisanenstreitkräfte gegenüber: Im Raum nördlich Udine und westlich des Kanaltales die eher nicht-kommunistische Divisione Osoppo-Friuli, welche mit den deutschen Stellen Kontakt aufgenommen hatte und primär am deutschen Abzug interessiert war, sowie die eher kommunistische Divisione Garibaldi-Friuli, die Ende April ihre Kampftätigkeit zum Teil verstärkte. Östlich des Kanaltales und weit bis in den Süden (Tarnovaner Wald, Aidussina) ausgreifend befanden sich ursprünglich die eher kommunistischen italienischen Partisaneneinheiten der Divisione Garibaldi-Natisone, der Brigata Garibaldi-Fontanot und der Brigata Garibaldi-Trieste, ferner die eher kommunistischen jugoslawischen Partisaneneinheiten des IX. Partisanenkorps. Die italienisch-kommunistischen Einheiten der Natisone, Fontanot und Trieste aber hatten sich schon am 7. November 1944 dem IX. Jugoslawischen Partisanenkorps unterstellt, wobei die Autorität der sowjetischen Militärmission von Oberstleutnant Ribaćenko beim Obersten Slowenischen Kommando nicht ohne Bedeutung gewesen sein dürfte. Am 24. Dezember 1944 waren alle diese italienisch-kommunistischen Partisaneneinheiten über den Isonzo nach Osten bis in den Raum Laibach abgezogen worden, wo sie unter schweren Verlusten bis zum Ende der Kämpfe um Laibach verblieben. Von britischer Seite wurde dieser Abzug der italienisch-kommunistischen Partisanen so gedeutet, daß die jugoslawische Führung bei ihren territorialen Besetzungsmaßnahmen, die sich auf ganz Friaul bis zum Tagliamento erstreckten, durch kritisierende und opponierende italienische Genossen möglichst wenig gestört sein wollte?.

Der weitere jugoslawische Vormarsch im Raum Triest und in Julisch Venezien dürfte genau den Plänen entsprochen haben, die im Februar 1945 zwischen Marschall Tito und Marschall Tolbuchin beschlossen worden waren: Während die Hauptmasse der 4. Jugoslawischen Armee möglichst rasch nach Triest vorstiẹ, bekam das IX. Jugoslawische Partisanenkorps den Befehl, den größeren Teil seiner Einheiten gleichfalls zur Eroberung von Triest und Görz in Marsch zu setzen und den kleineren Teil für den folgenden Einmarsch in Kärnten im Raum Tarvis - Ratschach bereitzuhalten. Als nun am Nachmittag des 1. Mai die Vorausabteilungen der 2. Neuseeländischen Division (Mj.Gen. Freyberg) ${ }^{10}$ bei Monfalcone am weiteren Vormarsch in Richtung Triest gehindert wurden, befand sich die Stadt nahezu vollständig in jugoslawischer Hand, doch eine ganze Reihe von deutschen Widerstandsnestern (vor allem MarineFlak und Reste der 188. Gebirgsdivision, nämlich die meisten Rekrutenkompanien) kapitulierte nicht vor den jugoslawischen Streitkräften. Die nicht-kommunistische italienische Freiheitsbewegung des Comitato di liberazione nazionale di Trieste hatte 
schon am 30. April versucht, die Verwaltung der Stadt zu übernehmen und mit den restlichen deutschen Kräften zu einer Übereinkunft zu kommen, wurde aber dann von den kommunistischen italienischen und slowenischen Gruppierungen und dem Stadtkommando Triest der 4. Jugoslawischen Armee aus dem politischen Spiel gedrängt. Ebenso nachdrücklich wurden die am 2. Mai zögernd in Triest einrückenden neuseeländischen Einheiten von jeder politischen und militärischen Aktivität abgehalten. Es hat also den Anschein, daß das so dynamische, sichelförmig weit nach Westen ausgreifende jugoslawische Besetzungskonzept nicht nur auf Triest, sondern auch auf Klagenfurt zielte, ehe man daran ging, von jugoslawischer Seite aus die militärischen Probleme Agram und Laibach zu lösen. Dieses weit ausholende Umgehungskonzept wird um so deutlicher, wenn man bedenkt, daß Triest am 1. Mai in jugoslawischer Hand war, die Ausrufung der Volksrepublik Slowenien und eine slowenische Regierungsbildung am 5. Mai in Aidussina erfolgten und Laibach erst am 12. Mai in jugoslawische Hand fiel. Man wird daher für die jugoslawisch-sowjetische Konzeption der Endphase im Alpen-Adria-Raum primär die konsequente politische Motivation territorialen Gewinnes annehmen müssen an Stelle einer militärischen Motivation der möglichst raschen Kampfeinstellung.

Globocnik hatte sich mit seinen Kräften zu einem Zeitpunkt nach Nordwesten zurückgezogen, zu dem aus Venezien auch ununterbrochen Reste der Heeresgruppe $C$ in Richtung auf Innichen, Kötschach-Mauthen und Villach zurückfluteten. Am 30. April abends verfügte Globocnik über eine lückenhafte Front von Cividale über Tarcento bis in die Enge von Venzone, die sogenannte Grüne Linie. Der zivile Verwaltungsapparat des Obersten Kommissars der Operationszone hatte sich schon in den letzten Apriltagen nach Kärnten zurückgezogen. Dieser Grünen Linie lagen auf britischer Seite vor allem Teile der zum V. Korps gehörenden 6. Panzerdivision gegenüber, während diverse Gruppen der Divisione Garibaldi-Friuli und des IX. Jugoslawischen Partisanenkorps versuchten, die deutschen Verbindungslinien nach Kärnten zu unterbrechen, was entlang den Hauptverkehrsadern aber nicht gelang. Schon Globocnik soll unter dem Einfluß seines Stabes danach getrachtet haben, mit britischen Stellen Kontakt aufzunehmen ${ }^{11}$. Schließlich verlegte er die militärische Führung nach Moggio Udinese und seinen Stab des Höheren SS- und Polizeiführers nach Arta Terme nördlich von Tolmezzo. Die Verbände von »Kosakenland in Norditalien“ und der Kaukasische Waffenverband entzogen sich zu diesem Zeitpunkt schon hartnäckig seiner Befehlskompetenz und traten in der Hauptmasse bereits am 30. April aus dem Sammelraum Tolmezzo den Rückzug über den Plöckenpaß an, zumal sich ihr Grundkonzept, Kampf gegen den Bolschewismus, auf die britische 8. Armee nicht anwenden ließ und auch schon seit Herbst 1944 Kontakte zu britischen Offizieren bei den italienischen Partisaneneinheiten gesucht worden waren ${ }^{12}$.

In Kärnten gab es Ende April/Anfang Mai die verschiedensten politischen Gruppierungen. Außer der politischen Führung der slowenischen Partisanenstreitkräfte waren alle Gruppen der Ansicht, es sei nützlich, wenn ein britisches Eindringen von Friaul nach Kärnten erfolge; nur über den gebotenen Zeitpunkt gab es Meinungsunterschiede. Von einer britischen Besetzung Kärntens erwartete man grundsätzlich eine gewisse Neutralisierung jugoslawischer Gebietsansprüche. In dieser Hinsicht stimmten auch einige Männer aus der engeren Umgebung Rainers mit den anderen Gruppen überein. Schließlich ließ sich auch Rainer selbst von der Richtigkeit dieser Konzeption überzeugen, nur hatte er eine andere Auffassung vom nützlichen Zeitpunkt als auch davon, inwieweit das Schicksal der Heeresgruppe E dabei berücksichtigt werden sollte ${ }^{13}$.

Wie war es aber bei den beiden betroffenen Heeresgruppen E und Süd selbst? Vielleicht hatte man bei der 2. Panzerarmee der Heeresgruppe Süd - die Heeresgruppe 
stand in der Steiermark und im südlichen Niederösterreich - wirklich schon am 29. oder 30. April von den Kapitulationsabsichten der Heeresgruppe $\mathrm{C}$ erfahren und deshalb versucht, Maßnahmen gegen ein rasches britisches und amerikanisches Vorgehen im Rücken der Heeresgruppe einzuleiten ${ }^{14}$. Wenn das zutrifft, so bestand der erste Schritt aber zweifellos nur in der Verlegung von Stäben nach Ostkärnten, was über die Verfügbarkeit zugehöriger Einheiten zum damaligen Zeitpunkt kaum mehr etwas aussagt. Die 2. Panzerarmee wurde jedenfalls erst am 7. Mai führungsmäßig der Heeresgruppe E unterstellt. Sicher ist, daß erst am 4. Mai Teile des Pz.Gren.Reg. 16 und der SS-Pz.Gren.-Div. zur Sperre des Seebergsattels und des Loiblpasses in den Karawanken freigesetzt werden konnten: „Unterstellung und Einzelheiten werden noch geklärt. Zurzeit Versammlung Nord Unterdrauburg. « ${ }^{15}$

Diese damit beabsichtigte Einnahme der wichtigsten Übergänge aus Slowenien nach Kärnten sollte aber offensichtlich nur verhindern, daß gemeinsam mit britischen Kräften aus Westen herankommende Partisanenstreitkräfte hier die Verbindung zwischen diesen beiden Ländern unterbrechen konnten.

Wann man sich bei der Heeresgruppe $E$ darüber im klaren war, daß diese durch die Kapitulation von Vietinghoff eine offene Westflanke hatte, scheint unklar. Generalmajor Schmidt-Richberg, Stabschef Löhrs, nennt den 2. Mai als den Tag, an dem die Heeresgruppe E von der Kapitulation erfuhr. Rainer aber wußte bereits am 29. April davon, und es erscheint bei der Intensität des Funkverkehrs zwischen Rainer und der Heeresgruppe $E$ eher erstaunlich, daß letztere nicht umgehend informiert worden wäre. Als Datum der offiziellen Benachrichtigung nennt Schmidt-Richberg erst den 4. Mai. Jedenfalls scheint ein Zusammenhang zwischen diesem Datum und dem späteren Zeitpunkt der eingeleiteten Gegenmaßnahmen der Heeresgruppe E zu bestehen, als da sind: Führungsmäßige Unterstellung der Division z. b. V. 438 (Generalleutnant Noeldechen) in Kärnten, Verlegung des Generalkommandos XXXIV (General der Flieger Felmy) aus Laibach nach Villach und des Generalkommandos LXIX (Generalleutnant Auleb) aus Agram nach Tanzenberg bei Klagenfurt. In Kärntner Berichten werden die beiden Generalkommandos zum ersten Mal am 7. Mai (Auleb) und am 8. Mai (Felmy) erwähnt, Schmidt-Richberg nennt kein konkretes Datum für die Verlegung ${ }^{16}$.

Mittlerweile versuchten verschiedene Widerstandsgruppen in Kärnten, Einfluß auf die militärischen Strukturen zu nehmen. Die Gruppe Greßel-Santer beispielsweise wollte z. T. mit Hilfe des Bezirksführers der Feuerwehren im Reichsgau Kärnten immer mehr Ortsfeuerwehren in ihr Netz einbauen, zugleich hatte sie auch Zugang zu den örtlichen Polizeikräften in Klagenfurt und Villach gefunden. Querverbindungen ergaben sich zur Heeresstandortverwaltung der Wehrmacht in Klagenfurt, wo Oberstleutnant Stossier Verhandlungen mit Kärntner Partisanenstäben anknüpfte, in denen es stets um Aufschub militärischer Aktionen ging.

Ein weiterer Schritt war die Kontaktknüpfung zu Einheiten der Division z. b. V. 438, so z. B. zum Gebirgs-Jäger-Ersatz-Regiment 139 und zum I. Geb.Art.Reg. 112, zugleich auch zur Luftwaffe, nämlich zum Fliegerausbildungsregiment 14. In allen diesen Gruppen stimmte man darin überein, Kärnten nicht zum Kriegsschauplatz werden zu lassen, weiter wollte man das Einströmen von jugoslawischen Partisanen- und Armee-Einheiten verhindern, da man sich territorialer Forderungen ähnlich jener von 1918 gewiß war. Daher tendierte man allgemein zu einem möglichst schnellem Herbeirufen britischer Streitkräfte aus Friaul. Den Überlegungen dieser Gruppen lag also ein durchaus anderes Kapitulationskonzept zugrunde, als dies bei der militärischen Führung der Fall war. Zugleich erscheint das Schicksal der Heeresgruppe E aus diesen Überlegungen im wesentlichen ausgeklammert. 
Stab der Division z. b. V. 438 selbst Kontakt auf. Am 3. Mai wurde Generalleutnant Noeldechen informiert und seine Zustimmung für eine frühe Kapitulation gegenüber britischen und amerikanischen Einheiten gewonnen, die man damit rasch nach Kärnten ziehen wollte. Zugleich ergab sich damit aber für Noeldechen die Notwendigkeit, Rainer zu isolieren und nicht mehr mit ihm zusammenzuarbeiten, falls dieser die Kapitulation hinausschieben wollte.

Rainer hingegen hatte Kärnten und das nördliche Friaul schon am 29. April von der bevorstehenden Kapitulation der Heeresgruppe $C$ ausgenommen und versucht, vor den Wehrmachtsdienststellen eine neue Front südwestlich Kärntens aufzubauen. Ein entscheidender Akzent ergab sich dabei durch das Auftauchen des Kommandeurs der 10. SS-Panzerdivision, Brigadeführer Heinz Harmel, in Kärnten. Dieser war bereits 1938 dort tätig gewesen und hatte seine Familie im Lande. Wegen seines Ausbruches aus dem Kessel von Spremberg (1. Ukrainische Front, Konjew) war er von Schörner seines Kommandos enthoben und zur Führerreserve nach Traunstein geschickt worden. Dort wußte man nichts von seiner Absetzung und ließ ihn wieder gehen. Er suchte Oberstgruppenführer Hausser in Bad Tölz auf und ließ sich von diesem raten, nach Kärnten zu gehen. Er fuhr zum SS-Feldkommandostab Hallein und meldete sich dort zu Rainer in Klagenfurt ab. Dort traf er am 30. April ein ${ }^{17}$. Rainer, der Harmel mit großer Erleichterung begrüßte, machte ihm bei dieser ersten Besprechung Mitteilung von der bevorstehenden Kapitulation der Heeresgruppe C (2. Mai, 14 Uhr) und gab ihm den Befehl, einen höheren SS-Führer in Friaul abzulösen und dort eine neue Front aufzubauen.

Harmel wies darauf hin, daß er keinen Stab habe und über keine Kenntnisse der Verhältnisse in Friaul verfüge; Rainer bestand aber auf seinem Auftrag. Offensichtlich leitete er die Kompetenz dafür aus seiner Funktion als Reichsverteidigungskommissar ab; es gibt aber keine Anzeichen dafür, daß er für seine nachfolgenden Maßnahmen die Zustimmung des Wehrmachtsführungsstabes B oder der Heeresgruppe Süd wie auch der Heeresgruppe E einholte. Zugleich zeigte sich auch, wie schmal die Basis der nationalsozialistischen Verwaltung bereits geworden war. Rainer stellte Harmel nämlich Gauleiter-Stellvertreter Thimel als Ia in Aussicht und versprach ihm den Einsatz der SS-Junkerschule Klagenfurt-Lendorf, eines Polizeiregimentes sowie von Einheiten der Hitlerjugend. Ob mit dem Polizeiregiment das in Kärnten stehende 13. SSPolizeiregiment (Obstlt. Fleckner) oder die ohnehin in Friaul eingesetzte 24. SS-Freiwilligen-Gebirgsdivision Karstjäger gemeint war, blieb unklar ${ }^{18}$. Bezeichnend ist, daß Rainer laut Harmel auch die in Kärnten stehende Division z. b. V. 438 nicht erwähnte und auch keine Verbindung zwischen ihm und Generalleutnant Noeldechen herstellte. Auch von einer Verbindung zu Fleckner oder einem Unterstellungsverhältnis zur Heeresgruppe E war nicht die Rede. Die Generalkommandos Felmy und Auleb befanden sich noch nicht in Kärnten. Löhrs Stabschef Schmidt-Richberg berichtet zwar, daß »um die Monatswende die Südsteiermark und Kärnten der Heeresgruppe $E$ als rückwärtiges Gebiet zugewiesen wurden « ${ }^{19}$, zugleich aber beschränkte er selbst noch am 5. Mai in seinem Funkspruch dieses rückwärtige Gebiet auf Ostkärnten, dessen östlichster Teil wiederum der Heeresgruppe Süd (= Ostmark) zugewiesen wurde. Mit der Zuweisung Ostkärntens an die Heeresgruppe $\mathrm{E}$ aber war dieser zweifelsohne die Division z. b. V. 438 einsatzmäßig unterstellt. Auch die Verlegung der beiden erwähnten Generalkommandos der Heeresgruppe E nach Kärnten teilt Schmidt-Richberg ohne Zeitangabe mit ${ }^{20}$. Am Morgen des 6. Mai befand sich das Generalkommando Felmy sicher noch in Laibach ${ }^{21}$.

Bei den Maßnahmen am 30. April und 1. Mai handelte es sich also ausschließlich um Rainers persönliche Initiative, wobei er sich über die Stimmung des Großteils der Bevölkerung im Lande, die nichts mehr vom Krieg und vom Nationalsozialismus wissen 
wollte, ebenso hinwegsetzte wie über die Einstellung oppositioneller politischer Gruppen. Zugleich schätzte er aber bei der Auswahl der begrenzten, ihm noch zur Verfügung stehenden Mittel die Realisierbarkeit wieder sehr zutreffend ein. Er dürfte unter folgenden Aspekten gehandelt haben:

1. Keine Teilnahme an der Kapitulation der Heeresgruppe $C$, sondern Aufrechterhaltung der nationalsozialistischen Herrschaft, daher keine Kapitulation ohne Befehl von oben, auch wenn jugoslawische Territorialforderungen und Besetzungsabsichten einen raschen britischen Einmarsch geboten erscheinen ließen, wobei er anscheinend von einer jugoslawischen Übermacht in Julisch Venezien und Friaul ausging und vielleicht einen kombinierten britisch-jugoslawischen Einmarsch aus dieser Richtung voraussetzte.

2. Aufrechterhaltung des status quo in Kärnten als Rückzugs- und Versorgungsraum für die Heeresgruppe $\mathrm{E}$.

3. Daher Halten einer Front in Friaul und Fernhalten sowohl britischer als auch jugoslawischer Kräfte.

4. Von diesem Verhalten versprach sich Rainer vermutlich günstigere Bedingungen für die Heeresgruppe $E$, solange der bevorstehende allgemeine Kapitulationstermin und die allgemeinen Kapitulationsbedingungen dies nicht verhinderten.

Zum damaligen Zeitpunkt ging noch ein großer Teil der möglichen Munitions- und Bedarfsgüterversorgung für die Heeresgruppe $E$ aus Kärnten nach Slowenien und Kroatien, zugleich wurde der Großteil der Verwundeten im Lande untergebracht 22. Dieser Andrang von Verwundeten stieg binnen weniger Tage so an, daß Rainer am 29. April und 1. Mai zur Aufnahme von Verwundeten bei der Bevölkerung aufrief³. Erst um das Jahresende 1945 konnten die letzten Wehrmachtslazarette in Kärnten aufgelöst werden ${ }^{24}$.

Harmel meldete sich am 1. Mai bei Rainer zum Einsatz, wo er von Gauleiter-Stellvertreter Thimel erwartet wurde ${ }^{25}$. Thimel war von Rainer als Stabschef Harmels vorgesehen. Harmel erhielt nach eigener Darstellung von Rainer lediglich einen mündlichen Befehl folgenden Wortlautes: 1. Ablösung von Globocnik in Moggio Udinese. 2. Verteidigung der Südgrenze Kärntens um jeden Preis, vor allem Sperrung der vier wichtigen Gebirgsübergänge Plöckenpaß, Naßfeld-Paß, Sattel von Saifnitz-Tarvis, Wurzenpaß. Wenige Tage später erinnerte Rainer an den Kärntner Abwehrkampf nach dem Ersten Weltkrieg, und Harmel gegenüber erwähnte er die Aufgabe der Kärntner Freiwilligen Schützen, die 1915 die Grenze gegen Italien halten mußten. 3. Im Falle einer Kapitulation sollte Harmel den britischen Verhandlungspartner drängen, möglichst schnell in Kärnten einzumarschieren und so weit wie möglich nach Osten vorzustoßen.

Von den sich in Kärnten bildenden Widerstandsgruppen war die Ankunft Harmels nicht eindeutig wahrgenommen worden. In den wenigen Berichten taucht »ein SSGeneral« auf, einige setzten seine Ankunft mit dem Eintreffen einer ganzen SS-Division im Norden Kärntens gleich ${ }^{26}$. Beim Kommando der Division z. b. V. 438 hatte man mittlerweile den Begriff »Kampfgruppe Globocnik« für die Verbände in Friaul geprägt und verwendete diesen Namen auch noch, als dieser Sperrverband längst nicht mehr von Globocnik geführt wurde ${ }^{27}$. Bei der Division wußte man also von Harmels Ankunft und von der Aufgabe, die Rainer ihm zugewiesen hatte.

Harmel erreichte, wie er selbst berichtet, in der Nacht vom 1. zum 2. Mai Moggio Udinese. Globocnik sei sofort nach seinem Eintreffen ohne Einweisung abgefahren. Er war in den folgenden Tagen in Klagenfurt, ging dann aber zu seinem Kommandostab nach Arta, um von dort am Rückzug über den Plöckenpaß teilzunehmen. Thimel wiederum fuhr mehrmals zwischen Moggio, Klagenfurt und dem Volkssturmkommando am Plöckenpaß hin und her, da Harmel sich seinen Stab aus Offizieren zu- 
rückgehender Einheiten gebildet hatte. Unklar bleibt, ob Rainer an Globocniks militärischen Fähigkeiten zweifelte oder ob er ihn als Stärkung seines Sicherheitsapparates in Klagenfurt haben wollte, womit sich Globocnik aber im Kompetenzbereich Röseners befand. Harmel versuchte, die durch das Kanaltal zurückziehenden Verbände neu zu ordnen, zu versorgen und in eine Front einzubauen. Die äußersten Positionen, nämlich der Plöckenpaß und der Wurzenpaß, wurden dabei aufgegeben, da es nicht gelang, Verbindung zu halten. Der Predilpaß - der zweite Zugang zu Tarvis wurde durch Kräfte der Ordnungspolizei aus Triest gegen jugoslawische Verbände gesperrt. Nach Harmels Schätzung standen ihm in diesen Tagen insgesamt rund 40000 Mann zur Verfügung. Die gegnerische Luftwaffe habe ständig beobachtet, aber kaum geschossen. Am 3. Mai läuteten überall die Kirchenglocken, weil die einheimische Bevölkerung an das Ende der Kampfhandlungen glaubte. Die deutschen Truppenführer machten auf Befehl Harmels den Ortsgeistlichen und den Bürgermeistern klar, daß es nicht so sei.

Die britische Seite verzögerte laut Darstellung Harmels ihren Vormarsch auffallend. Erst am 5. Mai wurde eine Melderrolle abgeworfen, die die Aufforderung zur bedingungslosen Kapitulation enthielt und in der widrigenfalls mit dem totalen Einsatz aller Luftstreitkräfte gedroht wurde. Harmel schickte einen Parlamentär mit einer aus vier Punkten bestehenden Antwort zu den Briten: 1. Die Drohung mit der alliierten Luftwaffe sei nicht eindrucksvoll. 2. Weder in der britischen noch in der US-Armee sei es üblich, ohne Befehl zu kapitulieren. 3. Er, Harmel, habe keinen solchen Befehl. 4. Er sei jedoch bereit, über eine $W$ affenruhe zu verhandeln, würde aber bei eindeutig erkannten britischen Bereitstellungen ohne Gegenangriff schießen, selbst wenn die Briten ihrerseits nicht schössen. Vom Stab der 6th Armoured Division wurde ein Captain als Unterhändler entsandt, doch verlangte Harmel, um Zeit zu gewinnen, einen ihm ranggleichen Unterhändler. Zugleich räumt Harmel Moggio Udinese und zog sich in mehreren Etappen, die stets wenige Kilometer umfaßten, unter geringfügigen Artillerieduellen zurück.

Das auffallende britische Zögern ist wohl kaum mit mangelhafter Aufklärung, Angst vor einer utopischen Alpenfestung oder hartem deutschen Widerstand zu erklären. Auch der von der Schlacht von Arnheim herrührende Respekt vor Harmel auf seiten der Alliierten reicht als Erklärungsgrund wohl nicht aus. Vielmehr lagen dem britischen Zögern politische Überlegungen zugrunde, vor allem die Diskussion um die Besetzung von Julisch Venezien und Friaul. Deshalb hatte das alliierte Mittelmeerkommando schon die Teilung von Julisch Venezien vorgeschlagen, da man beim Beharren auf der geplanten angloamerikanischen Militärverwaltung in dem ganzen Gebiet einschließlich Triest und Istrien - Gewalt anwenden müsse ${ }^{28}$. Truman hatte zuerst darauf beharrt, ganz Julisch Venezien ungeteilt in anglo-amerikanische Militär-Verwaltung zu nehmen, hatte dann aber am 30. April die Entscheidung wieder an den Vereinigten Generalstab delegiert und Feldmarschall Alexander angewiesen, vor einem weiteren Vormarsch Übereinstimmung mit den jugoslawischen Stellen herbeizuführen. Marschall Tito wiederum vertrat Alexander gegenüber den Standpunkt, daß auf Grund der großen Erfolge der jugoslawischen Streitkräfte das gesamte Gebiet von der Isonzomündung bis Tarvis als jugoslawisches Operations- und Verwaltungsgebiet anzusehen sei, so daß der britischen Seite immer deutlicher bewußt wurde, wie sehr der sichere Besitz von Triest und Julisch Venezien Voraussetzung für die Inbesitznahme der britischen Besatzungszone in Österreich war. Erst als Tito Alexander am 6. Mai mitteilte, die Pariser Friedenskonferenz habe nach dem Ersten Weltkrieg Julisch Venezien zu Unrecht an Italien gegeben, kam es am 7. Mai zu einem beschleunigten britischen Vorrücken in Richtung Kärnten ${ }^{29}$.

Das britische V. Korps gliederte nun seine bisher gedrängte Staffelung um: Die 6th 
Armoured Division (Mj. Gen. Murray) nahm weiterhin die Spitze im Kanaltal, im Raum Carnia schwenkte die 78th Infantry Division (Mj. Gen. Arbuthnott) nach Westen ab, im Raum Tolmezzo marschierte die 36th Infantry Brigade (Brig. Musson) über den Plöckenpaß. Obwohl die Kosaken- und Kaukasier-Einheiten schon abgezogen waren, erreichten die Vorausabteilungen der Brigade erst am 7. Mai die Paßhöhe. Zur gleichen Zeit hatte die 4. Jugoslawische Armee die zweite Phase ihrer Bewegungen eingeleitet: Motorisierte Einheiten stießen im Isonzotal nach Norden vor und suchten den Kontakt mit den bereitgestellten Einheiten des IX. Partisanenkorps zum Übergang über den Passo di Moistrocca in Richtung Wurzenpaß. Andere Einheiten der 4. Jugoslawischen Armee gingen im Windschatten der 6th Armoured Division vor.

Innerhalb des Sperrverbandes Harmel hatte sich im Abschnitt Pontebba-Tarvis immer deutlicher eine gewisse Rückgratfunktion der 24. SS-Freiwilligen-Gebirgsdivision Karstjäger und des 10 . SS-Polizeiregimentes gezeigt. Verbindung zum Wehrmachtsführungsstab B oder zur Heeresgruppe $E$ hat Harmel laut eigener Darstellung nie gehabt. Eine sporadische Verbindung zu Rainer hat aber offensichtlich bestanden, denn am 6. Mai informierte Rainer laut seinem eigenen Protokoll Generalfeldmarschall Kesselring darüber, daß von britischer Seite im Kanaltal die bedingungslose Kapitulation verlangt werde.

Mittlerweile hatten in Kärnten Vertreter der alten demokratischen Parteien gemeinsam mit Vertretern von Widerstandsgruppen unter Mitwirkung eines Teiles der NSDAP-Führung einen Aktionsausschuß gebildet, der die Einstellung der Kampfhandlungen gegen britische und amerikanische Kräfte sowie deren schnelle Herbeiholung forderte und den sofortigen Rücktritt Rainers verlangte. Der Aktionsausschuß konnte aber trotz seiner Funkverbindung nach Caserta und Udine keine Klarheit darüber gewinnen, ob britische Streitkräfte zu einem raschen Vorrücken bereit waren oder Rücksicht auf jugoslawische Bewegungen nehmen würden. Zusagen waren erst recht nicht zu erreichen. Die Division z. b. V. 438 hatte sich am 4. Mai auf die Seite des Aktionsausschusses gestellt und von Löhr die Erklärung Klagenfurts und Villachs zu offenen Städten erreicht. Dann tat der Divisionsstab folgendes: Die $^{\text {8. britische }}$ Armee stand an der Südwestgrenze des Landes zum Einmarsch bereit. Um eine kampflose Besetzung Kärntens so rasch als möglich herbeizuführen, wurden die eigenen Landesschützeneinheiten in diesen Abschnitten auf Kärntner bzw. Osttiroler Boden zurückgenommen und hatten Befehl, beim Vormarsch der alliierten Truppen sich kampflos zurückzuziehen oder die Waffen zu strecken.« ${ }^{30}$ In ähnlicher Weise ging die Division auch an der Südostgrenze Kärntens vor. Sie versuchte auch dort, Landesschützeneinheiten an die Grenze zurückzuziehen ${ }^{31}$. Dieser Vorgang spiegelt sich deutlich in der Reaktion des Kommandeurs der rückwärtigen Gebiete der Heeresgruppe E vom 6. Mai wieder, wobei sich zeigt, daß die Division wahrscheinlich das Gerücht aussprengen ließ, ihre Maßnahmen seien von Rainer befohlen oder daß die Mannschaften sich diese Maßnahmen selbst so erklärten. In dem betreffenden Funkspruch Röseners an Rainer heißt es: »In Veldes beim Landesschützenbataillon Zersetzungserscheinungen, Angehörige wollen nicht im slowenischen Raum, sondern erst wieder in den Karawanken kämpfen. Dem heute aufgetretenen Gerücht, daß alle deutschen Truppen bis 6. 5., 13.00 Uhr, die Karawankengrenze erreicht haben, müssen wir durch einen scharf gehaltenen Tagesbefehl des Kommandierenden Generals, rückwärtige Heeresgruppe $E$, entgegentreten. Für den Kommandeur der rückwärtigen Heeresgruppe E der Chef des Stabes, Obstlt. Hartmann. « ${ }^{32}$ Hinter dieser Maßnahme steckt anscheinend der eigenständige Versuch der Division z. b. V. 438, durch Stärkung der Positionen an der Landesgrenze ein weiteres Einsickern von jugoslawischen Partisanenstreitkräften zu verhindern. 
Rainer trat aber am 5. und 6. Mai trotz der an ihn gerichteten Forderungen des Aktionsausschusses nicht zurück. Auch der General der Wehrmachtordnungstruppe, Meißner, reagierte am 4. Mai auf die Verhältnisse an der italienischen Front. Sein Befehl lautete: "1. Die von Italien in den Westraum Kärntens eingetr. Angehörigen d. Wehrmachtstreifengruppen z.b. V. 3 und 6, Zugwachabteilung 516, Bahnhofswach-Streifenabteilungen 108 (Udine - Tarvis), Frontleitstelle 29 werden dem Major Gießen unterstellt. Dieser wiederum untersteht dem General der Wehrmachtordnungstruppen beim OB Südost (Oberkommando der Heeresgruppe E).« Die aus diesen Einheiten und den bisherigen Streifen des Wehrkreises XVIII/III im Raum Oberkärntens gebildete Wehrmachtstreifengruppe Gießen sollte vor allem die Gebiete nördlich des Plöckenpasses, des Grenzüberganges Tarvis und des Wurzenpasses unter Kontrolle halten ${ }^{33}$.

Rainers militärische Basis bestand zu diesem Zeitpunkt nur mehr aus einem Teil der Parteiorganisation und anderen Gruppen: Ein Teil der Junkerschule Lendorf wurde in Richtung Tarvis in Marsch gesetzt, am Wurzenpaß und in Tarvis waren mit Sicherheit bis zum 7. Mai Einheiten der Hitlerjugend zur Grenzsicherung eingesetzt ${ }^{34}$.

Zur gleichen Zeit trachteten der Wehrmachtsführungsstab A und die Reichsregierung Dönitz, bei Eisenhower einen Kapitulationstermin zu erreichen, der speziell die Situation der besonders exponierten Heeresgruppe E berücksichtigen sollte. Wenn Löhr wirklich mit Zustimmung von Dönitz am 5. Mai mit Feldmarschall Alexander Kontakt aufgenommen hat ${ }^{35}$, um ein möglichst weit nach Osten ausgreifendes Vorstoßen der Briten zu erreichen, so wäre unter diesem Gesichtspunkt auch seine Öffnung von Klagenfurt und Villach verständlich, ebenso seine wohlwollende Haltung gegenüber national-faschistischen slowenischen und kroatischen Bemühungen, ein möglichst weites britisches Vordringen nach Osten zu erreichen. Am 4. Mai waren als Abgesandte der kroatischen Nationalregierung von Ante Pavelic die beiden Verhandlungsbevollmächtigten Vrancic und Vrkljan von Agram nach Klagenfurt geflogen worden, um dann über Lienz und Innichen zur amerikanischen Front in Südtirol zu gelangen. Möglich ist es, daß die Heeresgruppe E oder auch das stellvertretende Generalkommando XVIII (General Ringel auf Hohenwerfen) ihre eigenen Kontaktversuche mit denen der Kroaten koordinierten ${ }^{36}$. Am 5. Mai sandte Rainer auf Drängen des Aktionsausschusses einen internierten britischen Offizier mit Begleitung zur amerikanischen Front in Richtung Salzburg, um ein rasches Vorrücken zu erreichen. Bemerkenswert erscheint, daß die Front in Friaul aus all diesen Kontaktversuchen ausgeklammert war. Es muß aber dann spätestens am 6. Mai vormittags eine sehr deutliche Ablehnung aller dieser Kontaktbemühungen von seiten Alexanders erfolgt sein, da erst mit diesem Tag nachdrückliche Planungen der Heeresgruppe E für die Stärkung der Italienfront einsetzten, obschon die Heeresgruppe sicherlich seit dem 29. April durch Rainer über die Entwicklung informiert war. Schmidt-Richberg gibt bezüglich dieser Maßnahmen bekanntlich keine Datumsangabe und berichtet auch nichts über eine Reaktion Alexanders auf die Kontaktversuche der Heeresgruppe. Vielleicht geht auch die eher einlenkende Haltung Rainers in Kärnten zu diesen Versuchen Löhrs parallel (am 4. und 5. Mai), bei Löhr wie bei Rainer zeigt sich am 6. Mai eine deutliche Verhärtung ihrer Position. Die Heeresgruppe zeigt in ihren Entschlüssen die typische Sicht der "Dinge von oben«, ohne noch alle die Realisierung bedingenden Faktoren einrechnen zu können. Rainers Maßnahmen stehen vor dem Hintergrund völliger Kriegsmüdigkeit der Bevölkerung und der Flüchtlingsmassen sowie zunehmender Zwangsmaßnahmen. Beide scheinen nunmehr unter Berücksichtigung militärischer Aspekte das Standhalten im Südosten gegen den außerordentlich starken jugoslawischen Druck der 1. und 2. Jugoslawischen Armee im Raum Agram, der 3. Jugoslawi- 
schen Armee im Raum Varazdin und der 4. Jugoslawischen Armee südlich Laibach für ebenso wichtig gehalten zu haben wie die Aufrechterhaltung der Verhältnisse in Kärnten als Auffangbereich für die Heeresgruppen E und Ostmark (zuvor Süd) bei Abschirmung gegen den britischen Vormarsch aus Südwesten, den man zuvor gesucht hatte.

Über eine Besprechung mit dem OB Süd, Kesselring, am 6. Mai in Graz, bei der Rainer nicht anwesend war, teilte Löhr Rainer mit: "Aus mir vorgelegten Meldungen sehe ich, daß ein englischer Offizier persönlich die Übergabe des Flugplatzes Zeltweg bei Judenburg verlangt hat. Dies gibt mir Veranlassung zu betonen, daß ich weder an Kapitulation noch an Übergabe militärischer Einrichtungen denke, solange nicht von vorgesetzter Stelle in dieser Richtung Befehle ergehen. Sollten sich im dortigen Bereich derartige Vorkommnisse ereignen, sind die betreffenden Offiziere General d. Fl. Felmy, komm. Gen. XXXIV, AK. Laibach, zuzuleiten. « ${ }^{37}$

Am späten Abend des gleichen Tages, nach der Besprechung mit Kesselring, befahl Löhr entscheidende Maßnahmen für die Südwestfront: »Befehlsführung in Kärnten:

1. Zur einheitlichen und straffen Befehlsführung im Gau Kärnten wird Phillipp in Westkärnten und Jochen in Ostkärnten eingesetzt.

2. Abschnittsgrenze zwischen Phillipp und Jochen: Tamsweg (69) - Ostende Ossiachersee - Mittagskogel.

3. Abschnittsgrenze zwischen Jochen und 2. Panzerarmee: Kamm der Saualpe Griffen (69) - Völkermarkt (69) - Lauf der Drau bis Gurkmündung sudwestlich Aich (10 ostsüdost Klagenfurt Haselberg) - 750,8 S (Rabenberg) - 15 SSW.

4. Territorialgrenze zu Korps Rösener: Kärntner Südgrenze 19405.«

Den beiden Generalkommandos wurden durch diesen Befehl einsatzmäßig alle Wehrmachtsteile in Kärnten unterstellt, die vollziehende Gewalt blieb beim Gauleiter und Reichsverteidigungskommissar, dem auch die Aufrechterhaltung von Ruhe und Ordnung übertragen war. Der Auftrag an die beiden Generalkommandos lautete, Westkärnten bzw. Ostkärnten mit Schwerpunkt an den südlichen Gebirgshängen durch Sperre und Verteidigung der Übergangsstellen zu sichern und dabei aus dem Raum Tarvis bei überlegenem Feinddruck hinhaltend kämpfend gegen Villach auszuweichen. Gegen Alliierte war der Kampf ausschließlich außerhalb der Orte vorgesehen. Der Befehl endet mit der Weisung: "8) Gen. Kdos. melden Befehlsübernahme und Gefechtsstände bis 8. 5. 9) NaFü stellt Draht- und Funkverbindung her. Alberich Ia 2712 gKdos 6. 5. 45. ${ }^{38}$ (»Alberich « ist der zu diesem Zeitpunkt bereits verwendete Deckname für Schmidt-Richberg bzw. dessen Stab). Dieser Befehl leitete offensichtlich erst die bereits mehrfach erwähnte Verlegung der beiden Generalkommandos Felmy und Auleb ein, so daß "Phillipp« und "Jochen« deren Decknamen wären, wofür es noch einen Hinweis gibt: Der Befehl berücksichtigte Harmels Position nicht, wendete sich aber auch deutlich gegen die Division z.b.V. 438 und stellte die Befehlsstrukturen und Herrschaftsverhältnisse in Kärnten wieder her, wie sie de facto überhaupt nicht mehr bestanden. Auch Rainer war sich trotz der Aktivitäten, die er vorhatte, bewußt, daß er nur mehr über Rumpfstrukturen verfügte. Offensichtlich sah er sich konsequent als Teil dieser militärischen Über-Strukturen, die nicht mehr in ausreichendem Maße mit der Realität in Einklang zu bringen waren. Bei Rainers Telephongespräch mit dem Stab Kesselrings am späten Abend des 6. Mai ging es jedoch um ganz konkrete Kapitulationsgegebenheiten und nicht darum, daß Rainer nun erst Klarheit über die wirkliche militärische Lage gewinnen konnte. Bei dem Gespräch erfuhr er, daß Kärnten von der Kapitulation der Heeresgruppe West (Ostgrenze Nordtirols) nicht betroffen sei.

Am 7. Mai vormittags hatte sich das Generalkommando Auleb nach der Verlegung aus dem Raum Agram in Tanzenberg eingerichtet und informierte um 11 Uhr den 
Kärntner Aktionsauschuß über Piesch, daß ein weiterer Funkverkehr mit britischen (und vielleicht auch jugoslawischen) Stellen in Italien unterbleiben müsse, da sich noch 150000 Mann der Heeresgruppe E außerhalb der Reichsgrenzen befänden. Im Verlaufe des gleichen Tages erfuhren dann der Wehrmachtführungsstab B und der OB Süd, daß Jodl bei Eisenhower keinen späteren Kapitulationstermin erreicht habe und auch der Truppe nach Einstellung der Kampfhandlungen keine Bewegungsfreiheit erlaubt worden sei. Beim OB Süd (Kesselring) kam der im Auftrag von Dönitz durch Keitel gezeichnete Befehl an, welcher schnellstens an die Heeresgruppen Ostmark und $E$ weitergeleitet werden sollte. In diesem wurde die beschleunigte Rückführung der Verbände nach Westen und die Übergabe an Amerikaner und Briten befohlen $^{39}$. Rainer befand sich zu diesem Zeitpunkt bei Kesselring in Graz und wurde daher unmittelbar über den endgültigen und allgemeinen Kapitulationsbefehl und seine Modalitäten informiert. Auch der Bericht seines Kontroll-Begleiters, des Aktionsausschuß-Mitglieds Tauschitz, hält fest, daß Rainer von dem Kapitulationsbefehl erfuhr. Damit war aber die Italienfront bedeutungslos geworden.

Folgende Ereignisse stehen mit dem obigen in engem Zusammenhang: Nun erst, am Nachmittag des 7. Mai, flog General der Flieger Felmy von Laibach nach Saifnitz, um Harmel den Kapitulationsbefehl zu überbringen. Rainer selbst trat am Abend des 7. Mai in Klagenfurt zurück, da er nun auch innerhalb des militärischen Apparates keine Funktion mehr hatte. Harmel aber kam nun dem Befehl Rainers vom 1. Mai nach: Er traf sich am Abend des 7. Mai mit dem Kommandeur der 6th Armoured Division, Mj. Gen. Horatius Murray, im Zollhaus von Thörl bei Tarvis und handelte mit ihm den frühestmöglichen Übergabetermin aus, zu dem der britische Offizier bereit war: den 8. Mai, 7.30 Uhr ${ }^{40}$. Auleb und Felmy erwarteten am 8. Mai um 12 Uhr Mittag Murray beim Kommando der Division z. b. V. 438 in Klagenfurt und führten die formelle Übergabe durch.

Bei der Besprechung Löhrs mit Kesselring in Graz war nach der Schilderung Schmidt-Richbergs durch den Generalfeldmarschall auch die Frage angeschnitten worden, ob die Heeresgruppe E etwa ohne Rücksicht auf einen allgemeinen Kapitulationstermin weiterkämpfen solle, um alle ihre Einheiten auf österreichisches Staatsgebiet zu bringen, doch habe sich Löhr nach reiflicher Überlegung dagegen ausgesprochen; dies vor allem mit Rücksicht auf den völkerrechtlichen Aspekt und die voraussehbare britische Haltung zu einem solchen Vorgehen ${ }^{41}$. Trotzdem wurde eine Entscheidung für das Weiterkämpfen über den Kapitulationstermin hinaus teilweise auf Divisions- und Bataillonsebene gefällt. Auch die kroatische Nationalarmee erzwang sich kämpfend aus dem Raum Unterdrauburg den Grenzübertritt in Richtung Bleiburg. Das alles führte nicht nur dazu, daß die Kämpfe im nordslowenischen Raum noch lange anhielten, sondern auch dazu, daß sie in Kärnten am 15. Mai endeten. Von politischer Bedeutung wurden diese Ereignisse dadurch, daß die jugoslawische Seite in Julisch Venezien wie auch in Kärnten mit der territorialen Angliederung groBer Teile des Landes an Jugoslawien rechnete. Die wirkungsvolle jugoslawische militärische Besetzung Südkärntens verzögerte sich aber durch das Andauern der Kampfhandlungen beträchtlich und gab daher in Julisch Venezien und in Kärnten den westlichen Alliierten die Zeit, günstigere militärische Positionen und eindeutige politische Standpunkte zu beziehen. 
1 E. Schmidt-Richberg: Der Endkampf auf dem Balkan. Die Operationen der Heeresgruppe E von Griechenland bis zu den Alpen. Heidelberg 1955 (= Die Wehrmacht im Kampf, Bd 5.), S. 135.

2 A. Walzl: Kärnten 1945. Klagenfurt 1985, S. 183.

3 Mitteilungen vom 9. 8. 1983 von Herrn E. Lerch an den Vf. Lerch war damals Leiter des persönlichen Büros Globocniks in Triest.

4 Vgl. M. Rauchensteiner: Krieg in Osterreich 1945. Wien 1970, S. 77.

5 In einem Vermerk auf dem Formular ist festgehalten, daß Rainer am 29. April mit einem Oberst Hessel telephonierte (im Besitz des Vf.)

- Funkspruch Heeresgruppe E (Schmidt-Richberg) vom 4. Mai 1945 an Stv. GK XVIII uber die vorbereitete Lahmung der Nachrichtenanlagen (im Besitz des Vf.)

7 So H. Jacobsen: 1939-1945. Der Zweite Weltkrieg in Chronik und Dokumenten. Darmstadt 1959, S. 535.

- Wiedergabe einer Drahtfunkansprache Rainers vom 3. Mai 1945 in *Karntner Zeitung* vom 4. Mai.

9 Kriegstagebuch der Divisione Garibaldi-Natisone. Archiv der Deputazione regionale per la storia del movimento di liberazione italiano nel Friuli - Venezia Giulia, Trieste, busta XIII, doc. 1505; C. R. S. Harries: Allied Military Administration of Italy 1943-1945. London 1957, S. 336.

10 Botschaft des Comitato di Liberazione nazionale an das Kommando der 2. Neuseelăndischen Division vom 5. 5. 1945. Archiv der Deputazione regionale (wie Anm. 9), busta LX, doc. 752.

11 Mitteilung Lerch (wie Anm. 3).

12 Nichts zu tun hat $\bowtie$ Kosakenland in Norditalien $\star$ mit dem XV. Kosaken-Kavallerie-Korps (Generalleutnant v. Pannwitz) das - zur Heeresgruppe E gehorig - weit im Osten stand, schließlich aber auch in Kurnten eintraf.

13 Walzl (wie Anm. 2), S. $127 \mathrm{ff}$

14 Bericht General de Angelis, Heeresgeschichtliches Museum Wien. Militarwissenschaftliches Institut. Abt. persönliche Berichte. Rauchensteiner (wie Anm. 4), leitet daraus die sofortige wirkungsvolle Verlagerung von Einheiten unter dem Gen. Kdo. XXII. Geb. K. ab.

15 Fernschreiben Heeresgruppe E an Stv. Gen. Kdo. XVIII vom 4. Mai 1945 (im Besitz des Vf.)

16 So im handgeschriebenen (ältesten) Bericht des Aktionsausschuß-Vorsitzenden und prov. Landeshauptmanns Hans Piesch und im Bericht von Obstlt. Payer vom Stab der Division z. b. V. 438 (im Besitz des Vf.)

17 Die folgenden Angaben gehen - wenn nicht anders vermerkt - auf ein Gespräch des Autors mit Herrn Harmel am 25. 5. 1985 zuruck.

18 Zur Karstjägerdivision vgl. Walzl (wie Anm. 2), S. 103 f.

19 Schmidt-Richberg (wie Anm. 1), S. 140.

20 Ebd.

21 Funkspruch Lohrs an Rainer, in Klagenfurt angekommen am 6. Mai, $10.00 \mathrm{Uhr}$ (im Besitz des Vf.)

22 Schmidt-Richberg (wie Anm. 1), S. 140.

23 „Kärntner Zeitung vom 1. 5. 1945 (Drahtfunkansprache Rainers v. 29. April).

24 Sitzungsprotokoll der provisorischen Kärntner Landesregierung vom 3. 10. 1945. Kärntner Landesarchiv, Sammelarchiv, Klagenfurt.

$25 \mathrm{Vgl}$. die teilweise wiedergegebene Darstellung bei M. Minyon: No Hope, No Orders, No Surrender, in: The Times vom 29. 4. 1985, S. 10.

26 Gemeinsamer Bericht Santer - Greßel uber die Arbeit der Widerstandsbewegung in Kămten (im Besitz des Vf.)

27 Bericht Obstlt. Payer (im Besitz des Vf.) Rauchensteiner (wie Anm. 4), S. 234, variiert die Bezeichnung: «Kampfgruppe Harmelx.

28 Harris (wie Anm. 9), S. 331.

29 Ebd., S. 336.

30 Bericht Payer (im Besizz des Vf.)

31 Sogenanntes Tagebuch Hütter, Teil Zusammenfassung, Kärntner Landesarchiv, Sammelarchiv, Klagenfurt.

32 Funkspruch Frontlagebericht Koruck Heeresgruppe E an Rainer vom 6. 5., angekommen 6.35 Uhr (im Besitz des Vf.)

33 Geheimschreiben Meißners an Reichsverteidigungskommissar Rainer vom 4. Mai, angekommen 5. Mai (im Besitz des Vf.)

34 Berichte an den Vf. von Herrn Dr. Benno Hundegger (vom 21. 12. 1984) und Herrn Albin Tschofenig (vom 25. 4. 1985). Der Widerstandskämpfer Tschofenig fuhr am 7. Mai mit einem vom Polizeikommissariat Villach zur Verfügung gestellten Dienstwagen auf den Wurzenpaß, überredete das Kommando der anruckenden Partisanenstreitkräfte zum Abwarten und bewog schließlich die in Stellung gegangenen Gruppen der Hitlerjugend zum Abzug. Unter dem Einfluß von Widerstandsgruppen war die Hitlerjugend in Villach mit Panzerfäusten ohne Zünder ausgerustet worden, um sie aus Kampfhandlungen herauszuhalten.

35 M. G. Steinert: Die 23 Tage der Regierung Dönitz. Düsseldorf, Wien 1967, S. 189 f.

36 E. Jareb und I. Omrčanin: The End of the Croatian Army at Bleiburg. London 1980, S. 71. G. Hoy und A. Legler: Schlußphase des Krieges im Bereich der Heeresgruppe E. In: Landesverteidigung, Wien 1962, Nr. 3. Rauchensteiner (wie Anm. 4), urteilt, daß General Ringel nach eigenen 
Angaben keinen Kontaktversuch in Lienz angestrebt haben kann, berucksichtigt aber nicht, daß dieser wüber Lienz « nach Brixen gemacht worden sein kann.

37 Funkspruch Löhrs an Rainer vom 6. Mai, angekommen 10.00 Uhr (im Besitz des Vf.) Die Zahlen hinter den Ortsnamen bezeichnen die Koordinaten auf der Generalstabskarte.

38 Fernschreiben Heeresgruppe $E$ an Rainer vom 6. 5. 1945, von der Fernschreibstelle angenommen 21.15 Uhr (im Besitz des Vf.)

39 Kriegstagebuch des Oberkommandos der Wehrmacht (Wehrmachtfuhrungsstab) 1940-1945. Geführt von H. Greiner und P. E. Schramm. Bd 4: 1. Januar 1944 - 22. Mai 1945. 2. Halbbd. Frankfurt a.M. 1979, S. 1675 ff. Steinert (wie Anm. 35), S. 384.

to Mitteilung von Herm Harmel an den Vf.

41 Bericht Payer (im Besitz des Vf.) 